\title{
SALUD PARA TODOS: CONCEPTUALIZACIÓN Y PERSPECTIVAS.
}

\author{
HEALTH FOR EVERYBODY: \\ CONCEPTUALIZATION AND PERSPECTIVES.
}

\author{
SAÚDE PARA TODOS: \\ CONCEPTUALIZAÇÃO E PERSPECTIVAS.
}

\section{Resumen}

El objetivo de este documento es estimular un análisis basado en la evidencia acerca de la salud para todos. Está destinado a profesionales quienes, desde sus propias prácticas, operacionalizan y regulan frecuentemente, la atención de salud. Se describen los antecedentes acerca de las diversas conceptualizaciones desarrolladas por instituciones e investigadores sobre el tema hasta arribar a la perspectiva actual. Entender esta perspectiva implicará incorporar los desafíos propios del pensamiento complejo, ya que en ella confluyen los requisitos de equidad, interdisciplinariedad, intersectorialidad,

Noemí Bordoni ${ }^{1}$ Aldo Squassi

${ }^{1}$ Profesora Emérita de la Universidad de Buenos Aires. Directora del Instituto de Investigaciones en Salud Pública Universidad de Buenos Aires. ${ }^{2}$ Profesor Titular regular de Odontología Preventiva y Comunitaria. Facultad de Odontología de la Universidad de

Buenos Aires. Vicedecano

Trabajo recibido: 04 de febrero 2019.

Aprobado: 20 de marzo transnacionalidad, las incertidumbres que crean las transformaciones de los contextos y las exigencias para cumplir eficazmente su cometido. Entre los actores en este escenario están las universidades quienes enfrentan la responsabilidad que les compete para dar cumplir con su misión social y cívica en la generación, difusión y aplicación de conocimientos tendientes a la salud para todos.

Palabras clave: Salud global- Salud para todos- misión universitaria.

\section{Abstract}

The objective of this document is to stimulate an evidence-based analysis on health for everybody. It is intended for professionals who, from their own practices, 
frequently operationalize and regulate health care. Diverse conceptualizations on the subject developed by institutions and researchers are described until we reach the current perspective. Understanding this perspective will involve incorporating the challenges inherent in complex thinking, since the requirements of equity, interdisciplinarity, intersectoriality, transnationality, the uncertainties created by the transformations of the contexts and the demands to effectively fulfill its mission converge in it. Among the actors in this scenario we find universities which face the responsibility to fulfill their social and civic mission in the generation, diffusion and application of knowledge aiming at health for everybody.

Key words: Global health- Health for everybody- University mission.

\section{Resumo}

O objetivo deste documento é o de estimular uma análise baseada na evidência da saúde para todos. Ele é destinado a profissionais que, a partir de suas próprias práticas, concretizam e regulam freqüentemente os cuidados de saúde. Descrevem-se os antecedentes sobre as várias conceituações desenvolvidas por instituições e pesquisadores acerca do assunto, para chegar na perspectiva de hoje. Compreender essa perspectiva vai envolver a inclusão dos desafios próprios do pensamento complexo, uma vez que aquela reúne os requisitos de equidade, interdisciplinaridade, intersetorialidade, transnacionalidade, as incertezas criada pelas transformações dos contextos e as demandas para atingir eficazmente o seu alvo. Entre os atores neste cenário acham-se as universidades que se deparam com sua responsabilidade para cumprir sua missão social e cívica na geração, difusão e aplicação de conhecimentos voltados para providenciar saúde para todos.

Palavras chave: Saúde global- Saúde para todos- missão universitária.

\section{Introducción}

El objetivo de este documento es estimular un análisis basado en la evidencia acerca de la salud para todos desde diferentes perspectivas. Está destinado a profesionales quienes, desde sus propias prácticas, operacionalizan y regulan frecuentemente la atención de salud. Entre los actores claves que encaran el tema no solo se encuentran los gobiernos sino que concurren también las universidades quienes enfrentan la responsabilidad que les compete para dar cumplir con su misión social y cívica en la generación, difusión y aplicación de conocimientos tendientes a la salud. El marco teórico -enunciado brevemente- sustentala perspectiva desde la cual se enfoca la evidencia seleccionada y las reflexiones que emergen de ella.

\section{Marco teórico}

Se enuncian algunos conceptos teóricos nucleares que enmarcan el abordaje de este documento.

- Poner la salud como objeto de estudio requiere aceptar que el sujeto ha organizado los datos a partir de sus modos generales (categorías) de pensar la realidad(1)

- La práctica científica consiste es una forma coherente y compleja de actividad humana cooperativa, establecida socialmente, cuyos practicantes han incorporado un saber que les permite conservar $\mathrm{y}$, a la vez, renovar el sentido de la práctica científica.(2)

- El razonamiento científico emplea reglas en su proceso interpretativo que lo diferencia del razonamiento cotidiano. Entre otras: la regla de la duda ilimitada, la regla de publicabilidad y la afirmación que la observación de los aspectos sociales de la organización y de la comunicación científica son insuficientes y que deben 
abordarse en forma disciplinada.(3)

- La traducción del conocimiento en acciones políticas o sanitarias consiste en tomar como objeto de estudio el proceso de construcción social de conocimientos preconstruidos.(4) Instala, por lo tanto, la reflexión sobre la dimensión ética..

- $\quad$ El impacto es el conjunto de cambios -favorables o no- que se producen y mantienen en la sociedad, la economía, la ciencia, la tecnología y el medio ambiente, los que introducen un valor agregado a los productos, servicios, procesos y tecnologías de salud. (5)

- El impacto de la marginación social sobre la salud es un fenómeno no solamente determinado por las condiciones de vida sino también por el modo de vida, el estilo de vida cuando se tiene la libertad para elegirlo y la calidad de vida en su condición objetivo/subjetiva.(6)

\section{CONCEPTUALIZACIÓN DE SALUD PARA TODOS}

\section{Análisis desde diferentes perspectivas}

A través del tiempo se han desarrollado una cantidad de centros académicos especialmente en Estados Unidos y Europa que abordaron la salud desde diferentes perspectivas.(7).La mayoría de esos centros se ubican en países de altos ingresos vinculados, frecuentemente, con otros de medios o bajos ingresos. El concepto de salud para todos parece todavía resultar indefinido al adjudicarle variadas concepciones $(8 ; 9 ; 10 ; 11)$ y muestra la necesidad de alcanzar una definición común corta, precisa y consensuada, incluyendo la aceptación por parte del público.

Koplan et al(8), integrantes del Consorcio de Universidades de Salud Global, señalaron que sin una definición aceptada de salud global, resultará difícil ponerse de acuerdo en lo que ella está tratando de lograr, cómo se hará y como se monitoreará su progreso. Esto resulta particularmente complejo debido a que las recientes crisis -cambio climático, económico, crisis alimentaria y energética- han acentuado las dificultades que enfrentan la teoría y la práctica en salud global. Estos autores establecieron diferencias entre las conceptualizaciones de salud global, salud internacional y salud pública. En algunos documentos, el término la salud global se utilizó como sinónimo de salud pública tradicional y las acciones que ella aplica.Pero la conceptualización de salud pública también ha transitado transformaciones. Se la interpretó como:

(a) un estado de completo bienestar físico, mental y social y no sólo como la ausencia de enfermedad (Constitución de la OMS, 1946, entrado en vigencia en 1948)

(b) en el marco del Desarrollo Humano se define como la capacidad y los recursos de cada persona para vivir, aprender, producir, participar, amar y conducir su existencia(12);

(c)el arte y la ciencia de prevenir la enfermedad, recuperar y promover la salud, a través de los esfuerzos organizados de la sociedad y lo que puede hacerse colectivamente para asegurar las condiciones para que las personas sean saludables (13);

(d) argumentos basados en la necesidad de abordar los determinantes sociales, económicos, ambientales y políticos subyacentes a la salud, con independencia de que el objetivo principal se plantee a nivel nacional o global, la salud pública sido definida como un"enfoque en la salud de la población de un país o de una comunidad específica" (14) Estaperspectiva es compartida a posteriori por Fried et al.(9) quienes plantearon que «la salud pública es la salud mundial para el bien público».

Otra perspectiva se plantea al definir salud internacional. El término salud internacional surgió con la creación de la Comisión de Salud Internacional en 1913 y con la creación del International Health Board y se destacó como disciplina académica en la segunda mitad del siglo XX. Este término se ha utilizado para referirse a «la participación de los países en la labor de las organizaciones internacionales como la OMS, por lo general a través de pequeños departamentos de salud" $(15 ; 16)$.La salud internacional se interpretó en estos casos como "centrada sobre los problemas de salud registrados en los países 
de bajos ingresos, especialmente sobre las enfermedades infecciosas y la salud maternoinfantil".

Merson et al(17) La definieron como «la aplicación de los principios de la salud pública a los problemas y desafíos que afectan a los países de bajos y medianos ingresos y para el complejo conjunto de fuerzas globales y locales que influyen en ellos».

Teruel(18) identificó cuatro áreas de interés para la salud internacional:

- $\quad$ aspectos relacionados, directa o indirectamente, con los problemas de salud o sea a las enfermedades en general;

- $\quad$ la forma como se desarrollan programas al servicio de los países;

- la mala utilización de los recursos existentes;

- actuación de todas las agenciasy organismos que se desarrollan en el campo de la asistencia y la cooperación internacional.

Koplan et al caracterizaron a la salud global como: "un área de investigación y práctica que ubica la prioridad en el mejoramiento y logro de la salud en equidad para todos los pueblos del mundo"(8). Esta definición resulta útil porque establece un amplio foco sobre mejoramiento de la salud en equidad.

Kickbush(19) definió salud global como responsable de: "los asuntos de salud que trascienden los limites nacionales y gubernamentales y convocan a las fuerzas globales para la acción". Esta definición plantea un amplio foco pero, según Beaglohole et al(20) resulta pasiva al convocar para la acción y omitir la necesidad de la investigación y de las acciones colaborativas que destacan Koplan et al. .

Convocatorias de la European Foundation Centre(21)fueron realizadas para lograr una aproximación continental sobre los problemas que concita la salud global y la considera como "una política prioritaria a través de todos los sectores y basada sobre bienes públicos globales". Documentos emitidos por el Gobierno de Gran Bretaña(22) se refirieron a la salud global como "un asunto de salud donde influyen determinantes, indefinidos $u$ obvios que exceden los límites territoriales y están más allá de la capacidad individual de los países y la intervención de las instituciones domésticas". Esta definición incluye importantes ideas (determinantes de salud, dimensiones territoriales, responsabilidades institucionales) pero no explicita los productos a alcanzar. Mac Farlane et al(7) completaron el concepto al identificar como productos a lograr: "el aumento mundial de salud, la reducción de disparidades y la protección contra las amenazas globales que no respetan limites nacionales".

En el 2014, durante el 53․ Consejo Directivo de la Organización Panamericana de la Salud/Organización Mundial de la Salud (OPS/OMS), los países de la Región de las Américas reafirmaron su compromiso con la Estrategia para el Acceso Universal a la Salud y la Cobertura Universal de Salud (Salud Universal)(23). En reuniones posteriores se introdujo la estrategia de educación interprofesional de salud, que refuerza el papel de las instituciones formadoras de recursos humanos en el cumplimiento de esos propósitos.

\section{Salud para todos y las categorías implícitas.}

La salud para todos es una manera de ver y abordar la salud como un bien público mundial, como un tema de justicia social y como un derecho universal. Para poder ubicar las inequidades y desigualdades de salud en el centro del debate político, es necesario desarrollar mejores vías para la evaluación. Teruel y Wegman desarrollaron una definición para salud global como "un nuevo enfoque dinámico, basado en el análisis de la diversidad y de las tendencias en salud ylas condiciones de vida de poblaciones y países, la influencia de las fuerzas políticas y socioeconómicas y el uso del conocimiento en la solución de los problemas identificados".(24)

El conocimiento complejo de la salud para todos exige adentrarse en la problemática de su construcción social, como un continuo en el mundo cultural. La adquisición del 
conocimiento complejo de la salud mantiene una naturaleza cultural. Definirla implica cumplir con una serie de variablesy de las categorías que contribuirán a la construcción de su identidad como tal.

La salud para todos:

- gira en torno a la equidad, la ética y el respeto a los derechos humanos fundamentales,

- analiza temas y riesgos nuevos y remergentes,

- $\quad$ privilegia el trabajo con grupos poblacionales en desventaja social,

- $\quad$ responde a los retos comunes con profundidad local y alcance global,

- $\quad$ promueve la participación ciudadana,

- impulsa la interdisciplinariedad y la intersectorialidad,

- vincula a agentes económicos, políticos y sociales, en búsqueda de la construcción de la intersubjetividad,

- $\quad$ traduce hallazgos a acciones concretas y cuantificables y

- enfatiza colaboraciones horizontales y procesos compartidos entre instituciones, países y regiones y vecindarios.

\section{Análisis de las categorías requeridas para la construcción de salud para todos}

\section{- $\quad$ Acerca de la equidad}

El requisito esencial en salud para todos es la equidad. En la búsqueda de un modelo para alcanzar para traducir la producción de conocimientos en acciones informadas en la evidencia científica contextualizada y tendiente a lograr la equidad en salud pueden reconocerse dos momentos: (a) definición de un modelo; (b) evaluación de su efectividad. Davison et al(25) en una revisión sistemática realizó un análisis crítico de los modelos existentes para relacionar conocimientos en intervenciones efectivas tendientes a superar las inequidades de salud global existentes en la actualidad. En su estudio realizado sobre un total de 689 publicaciones (PUBMED, PsychINFO, AMED, eBSCO, Google Scholars, revisión de referencias mencionadas, consulta de expertos) identificó 48 modelos empleados para evaluar la traducción del conocimiento en acciones efectivas (CEA) $\backslash$, tendientes a proveer equidad en poblaciones excluidas. Los resultados obtenidos mostraron que 8 modelos alcanzaban un score entre 8 y 10 puntos sobre un máximo de 12 puntos de equidad en salud. (FIGURA 1)

En todos los casos, el factor relacionado con la equidad más frecuentemente perdido fue: "las dificultades o la pérdida de la aproximación intersectorial".

FIGURA 1: CRITERIOS PARA EVALUAR EFECTIVIDAD DE MODELOS DE INVESTIGACION TRASLACIONAL Fuente: Davison et al, $2015^{25}$

(1) foco especifico, mención o consideración en términos de equidad, igualdad, justicia, grupos en desventaja o vulnerabilidad;

(2) conceptualización inclusiva del conocimientos que aseguren diferentes tipos de conocimientos y/o vías para alcanzar conocimientos basados en la evidencia;

(3) miembros de la comunidad representados y/o participantes en una parte explicita del modelo;

(4) debe ser sostenida la interacción entre disciplinas o sectores

(5) existir referencia especifica al contexto social, físico, político y/o económico en el proceso de generación y uso del conocimiento

(6) debe ser un modelo aplicado, proactivo o focalizado en la solución de problemas,

Las inequidades en salud son el resultado de un sistema complejo que funciona en el ámbito local, nacional y mundial y surgen fundamentalmente cuando no se abordan eficazy oportunamente las desigualdades evitables. Todas las sociedades tienen jerarquías sociales cuyos recursos sociales y económicos, incluidos el poder y el prestigio, están distribuidos de forma desigual. La distribución desigual de los recursos tiene un impacto en la libertad de las personas de llevar vidas que puedan valorar(26).A su vez, esto tiene 
un gran impacto en la salud y su distribución en la sociedad. En ambas situaciones, la pregunta es conocer cómo se originan estas causas. Es decir, ¿cuáles son las causas de las causas? Esto conduce a la búsqueda de factores determinantes sociales de la salud y de la equidad en salud.(27).

En la dimensión mundial, la globalización, los sistemas de salud, las zonas urbanas, el empleo y las condiciones laborales, la exclusión social, lasinequidades en cuestiones de género, así como la violencia y los conflictos, los alimentos y la nutrición y el medio ambiente son variables intervinientes en la salud de las poblaciones. Las altas tasas de enfermedades, particularmente enfermedades infecciosas y malnutrición que afectan a las personas pobres en los países de bajos y aún en los de medio ingreso reconocen como causas: (a) la falta de comida, (b) el agua contaminada, (c) los bajos niveles de saneamiento, (d) a incapacidad para desenvolverse en medios que involucran una exposición elevada a agentes infecciosos y (e) la falta de atención adecuada. En forma similar, se conocen ampliamente las causas de las enfermedades no transmisibles que representan la carga principal de enfermedad de las personas en el extremo más bajo del gradiente social en los países de ingresos medios y altos. Mediante el estudio sobre la carga de enfermedad mundial se identificó al bajo peso, al sobrepeso, al tabaquismo, al consumo de alcohol, a la violencia en todas sus expresiones, a la hipertensión y a la conducta sexual como las principales causas de morbilidad y mortalidad. (28).

La categoría de equidad requerida en la conceptualización de salud global-y obviamente en la dimensión de salud para todos- lleva implícita el desarrollo de las siguientes condiciones: (a)interdisciplinariedad,(b)intersectorialidad,(c)transnacionalidad/interregionalidad,(d) complementariedad/colaboratividad (e) contextualización para identificar inequidades y desigualdades.

\section{- $\quad$ Acerca de la interdisciplinariedad}

La condición de interdisciplinariedad se refiere a la necesidad de dar respuesta a la salud en tanto problemática social compleja y expresada en diferentes campos o componentes de la salud, en las dimensiones individual o colectiva. Entre otros focos de investigación y acción de interés que requieren el abordaje interdisciplinar pueden citarse: la comprensión y el abordaje de conductas sociales de violencia, la vinculación entre expresiones parciales del proceso de salud-enfermedad-atención-cuidados (componente mental, componente bucal, epidemias, etc) y factores relacionados con la pobreza, la relación entre el uso irracional de agroquímicos y la emergencia de enfermedades neurológicas.

La visión de salud exige analizar la causa y "la causa de las causas" y ello solo es posible desde la perspectiva de diferentes disciplinas y campos de expertez. Por lo tanto, el abordaje de esos conceptos deben plantearse desde la complejidad o a través de su estrategia metodológica: la interdisciplinariedad, que actúa como proyección pragmática a corto plazo para desarrollar un conocimiento sensible al valor epistémico de la complejidad.

El objetivo último de cualquier investigación interdisciplinaria es conocer una porción del mundo modelizado por un sistema complejo determinado, en este caso, la salud. Todas las categorías intervinientes en las intervenciones en busca de salud para todos sufren procesos de de-diferenciación, de-aislamiento e hibridación, poniendo en incertidumbre los límites y las funciones de los actores que participan en esos procesos. Las investigaciones interdisciplinarias tienen, en general, como propósitos:

a) dar respuesta a problemas estratégicos, prioritarios o que constituyan una demanda económica y social.

b) expandir la base científica, vinculado con la disponibilidad de recursos de diferente naturaleza y su articulación.

c) obtener economía de escala o

d) fortalecer alguno de los nodos estratégicos de una red. 
El abordaje desde la generación y aplicación de conocimientos y de la colaboración interprofesional constituyen un inteligente abordaje para encarar efectivamente esta vacancia.

\section{- $\quad$ Acerca de la intersectorialidad}

La intersectorialidad ha demostrado ser la condición más lábil al analizar la traducción de los conocimientos informados en la evidencia en acciones políticas o sanitarias. Sin embargo, al analizar "la causa de las causas" de esta debilidad, puede ubicarse la problemática en la endogamia de la academia en el cumplimiento de su misión social y cívica y la escasa vinculación entre el sistema educativo y el sistema de salud en los niveles de grado y posgrado.(29)

Desde hace más de 30 años se vienen desarrollando en América latina rigurosas experiencias de vinculación intersectorial. La Universidad de Buenos Aires la concretó en diferentes en escenarios vinculada con el sector salud y la comunidad (ONG, comunidades barriales). Experiencias destacables son las desarrolladas por la Facultad de Odontología, (30; 31; $32 ; 33,34,35,36,37$.) en áreas urbanas y rurales desde 1983, en el marco del PADAI (1984-1992), asociada en ocasiones con la Facultades de Agronomía, de Ciencias Exactas y Naturales, de Arquitectura, Diseño y Urbanismo y de Farmacia y Bioquímica. Otros equipos de las universidades de Bs As (CIRFS/FADU; 1982); de Córdoba (FM/1985); de Tucumán(Proyecto UNI/UNIR, 1986-1992); de Rosario (1988) y de Ministerios y Secretarias de salud (Proyectos San Pedro y Adolescencia (1990) generaron experiencias valiosas con repercusión y participación en proyectos internacionales (Proyecto REDES y Proyecto NORTE-SUR).

En el año 2010 la Global Independient Comission reconoció tres grandes reformas en educación superior en ciencias de la salud (38):

1. basada en la ciencia, lo que da lugar a la instalación de currículos científicos, expertez disciplinarias y se instala en las universidades.

2. basado en problemas, da lugar al aprendizaje basado en problemas, y se instala en centros académicos multicéntricos.

3. basado en sistemas, da lugar a la construcción de competencias en los niveles localglobal y se instala en la articulación de los sistemas de educación y de salud.

- $\quad$ Acerca de la transnacionalidad

La transnacionalidad se refiere a que la preocupación de la salud para todos se fija sobre las cuestiones que trascienden las fronteras nacionales a pesar de que sus efectos pueden experimentarse dentro de los países. Lograr la salud requiere acciones que requieren la participación de más de dos países, uno de ellos fuera de las organizaciones regionales tradicionales, requisito que excluye su interpretación como problema localizado o regional, mientras que, al mismo tiempo, suele sustentarse en las instituciones nacionales de salud pública. Teruel y Wegman(24)destacaron esta condición al conceptualizar la salud global. La reconocieron como la salud sin fronteras, como un área del conocimiento integral, interdisciplinaria, política y práctica tendiente a mejorar la salud individual y colectiva, alcanzar la equidad y contribuir al desarrollo global a través de alianzas transnacionales y colaboración intersectorial. Este atributo toma vigencia en el momento en que las migraciones y sus actores configuran un nuevo escenario para abordar la salud(39).

- Acerca de la colaboratividad/complementariedad

El análisis de la colaboratividad/complementariedad destaca la importancia crítica de la colaboración para hacer frente a: (a) los problemas de salud; (b) las cuestiones que tienen una multiplicidad de factores determinantes y (c) la disponibilidad de un complejo conjunto de instituciones que intentan participar colaborativamente en la búsqueda de soluciones.

El logro de colaboratividad requiere un proceso en el cual los vínculos son la base de su 
construcción. Esto requiere reconocer a los otros como interlocutores o socios, hecho que supone y genera aceptación; conocer lo que el otro hace, lo que supone y genera interés; co-laborar es decir prestar ayuda esporádica, lo que supone reciprocidad; cooperar, es decir compartir actividades, conocimientos y recursos, acciones que suponen y generansolidaridad y por fin, asociarse para sostener proyectos o iniciativas conjuntas, situaciones que suponen y generan confianza. (40)

- Acerca de la contextualización en busca de la equidad(41)

El empoderamiento también pretende ser uno de los productos de los procesos deliberativos intersectoriales y satisface uno de los requisitos reconocidos en la conceptualización de Salud Global. Se identificacomo la expansión de los activos y capacidades de los individuos o los colectivos para participar en, negociar con, influir sobre, controlar y tener instituciones que influyan en sus vidas. No se centra únicamente en características individuales sino que tiene en cuenta características estructurales que abarca grupos sociales enteros. Como la exclusión es multidimensional, los activos y capacidades requeridos para mitigarla también deben ser multidimensionales. (42)

Los activos pueden ser físicos o financieros y habilitan a la gente a resistir inconvenientes y a expandir su horizonte de decisiones. Las capacidades desarrolladas son inherentes a las personas y las habilitan para usar adecuadamente sus activos, tales como: la capacidad de organizarse o analizar las normas y redes de trabajo. Los elementos componentes del empoderamiento son: (a) acceso a la información, (b) inclusión y participación, (c) responsabilidad o rendición pública de cuentas y (d) capacidad organizativa local.

En ocasiones, racionalmente ejercidas y pertinentemente justificadas, pueden conducir a lograr el acceso a servicios básicos, a mejorar la gobernación local o nacional y a acceder a la justicia o a la ayuda legal. Los grupos sectoriales o extrasectoriales pueden ser actores sociales decisivos para la instalación y mantenimiento de programas de Salud Global.

\section{Acerca de la investigación traslacional}

Los requisitos asignados a la salud global implican la importancia de desarrollar investigación para lograr evidencia que sustenten la toma de decisiones políticas, especialmente las que ponen de relieve los efectos de factores determinantes transnacionales de la salud(42; 43). Las acciones resultantes hacen hincapié en el uso de esta información de manera constructiva en todos los países para alcanzar la equidad sanitaria. La promociónde la salud implica la importancia de utilizar una amplia gama de estrategias para mejorar la salud, incluyendo las dirigidas a los determinantes sociales, económicos, ambientales y políticos subyacentes de la salud..(44)

\section{Discusión de los alcances y las perspectivas}

a. Obstáculos y amenazas

En la Conferencia Mundial sobre la Ciencia (45)surgió la necesidad de establecer un nuevo acuerdo entre la ciencia y la sociedad. Los líderes de las ciencias naturales adoptaron los postulados que daban cuenta de la necesidad de la participación democrática, la integración y la ética. Este hecho inusual coincidió con una paulatina desacralización que la sociedad hizo de las ciencias y de los científicos, determinada por la educación científica impartida sin considerar la relevancia y pertinencia de la generación de los conocimientos.

Investigaciones universitariasafirman que, en ocasiones, es la propia comunidad científica la que, por hegemonías institucionales internas o por determinantes externos, obstaculiza el proceso de traducción entre la producción de conocimientos y la formulación de políticas. Los mismos criterios de evaluación establecidos hacia adentro de los sistemas de educación superior desalientan la producción de conocimientos estratégicos y con pertinencia social, especialmente útiles en circunstancias donde deben resolverse situaciones críticas como las que muestra la inequidad en salud. Puede argumentarse que, en tanto no se establezcan 
incentivos para las actividades de investigación y desarrollo con impacto social, resultará difícil que los investigadores se encuentren estimulados para buscar la vinculación con los formuladores y hacedores de políticas, quienes debieran ser los usuarios informados del conocimiento producido.Hacia el interior de las universidades se busca introducir indicadores de evaluación que den cuenta del impacto social de las investigaciones. (46; 47)

Asimismo, es necesario reconocer que la formulación de políticas es una actividad realizada en un sistema abierto y, por lo tanto, menos prescriptivo que la investigación. Es un proceso largo cuyo inicio es la formulación de la política que se continúa con la interpretación que se hace de esa decisión en cada escenario local $(5 ; 48)$. Al analizar la interfase entre el espacio de la ciencia y el de la política se evidencian variadas divergencias: (a) el ritmo de formación de la opinión pública resulta sometido a más imponderables que el mundo de la ciencia; (b) la complejidad del idioma técnico dificulta la instalación de los problemas abordados en la agenda social; (c) la ciencia requiere bases de datos empíricos mientras que la formulación de políticas requiere una base de valores.(48)

Woolf et al(49)plantean que la desconexión es particularmente frecuente en salud donde los agentes de cambio son quienes pueden hacer las mayores diferencias ya que las conductas de salud y las condiciones sociales y ambientales están regidas por personas no informadas. Describen que se requieren cuatro ingredientes para alcanzar éxito: (a) investigación que responda a las necesidades del usuario, (b) conocimiento del ambiente donde se toman decisiones, (c) compromiso entre los "asociados" y (d) comunicación estratégica.

Una paradoja generada por la indicialidad de los proyectos, o sea por su emplazamiento en un contexto de tiempo, de espacio y eventualmente de reglas tácitas -es decir su contingencia situacional y a su emplazamiento contextual- es la tardía legitimación para su traslación a decisiones político-sanitarias. Sin embargo, su impacto puede verificarse en la actualidad en muchas universidades, en las cuales se fueron generando las condiciones de interdisciplinariedad, intersectorialidad, transnacionalidad, colaboratividad y desarrollo de modelos que aplicaron el enfoque de riesgo en el nivel individual y el enfoque de vulnerabilidad al instalar las prácticas situadas en comunidades desprotegidas o excluidas. Todas ellas están hoy reconocidas como requisitos para avanzar en la teoría y la prácticas propias de la salud para todos.

\section{b. La responsabilidad social y cívica de las instituciones como fortaleza y oportunidad}

Las instituciones sean educativas o desalud deben investigar los asuntos que se afrontan en la toma de decisiones políticas con el propósito de regular la asimetría entre la investigación que se está realizando y las necesidades a las que debe darse respuestas, si es posible, en forma anticipatoria y desde un marco ético $(50 ; 51)$.

Una hipótesis de trabajo (42)es instalar la reflexión y la acción a partir de procesos deliberativos interactivos que aclaran objetivos, son inclusivos y transparentes, estimulan el diálogo, promueven el consenso e impactan sobre la decisión en sí misma. Estos procesos que implican un análisis técnico y la deliberación entre actores clave y público contrastan con la tradicional orientación vertical de las políticas. Los procesos deliberativos pueden ser convocados por los diferentes actores institucionales: universidades, entidades de I+D o de servicios científicos tecnológicos, entidades de desarrollo tecnológico, instituciones o agencias del Estado, proveedores, clientes, organizaciones superiores, organizaciones no gubernamentales y organizaciones sin fines de lucro, entidades intermedias del conocimiento, servicios científicos-tecnológicos, instituciones o agencias del Estado, proveedores, clientes, organizaciones superiores, consultoras, polo científico-productivo y grupos integradores. $(5,41)$

Para obtener impacto sobre el proceso de salud-enfermedad-atención-cuidadosen cada 
una de las dimensiones local/global basándose en la mejor evidencia disponible, deben preverse la siguiente secuencia de estrategias. (52)

- monitoreo del estado de salud para identificar los problemas de salud en los niveles locales,

- diagnóstico de los problemas identificados y de los riesgos potenciales en niveles regionales o globales,

- información, educación y empoderamiento de personas y comunidadessobre asuntos de salud,

- movilización del partenazgo comunitario para identificar y controlar riesgos y resolver los problemas potenciales,.

- desarrollo de políticas y planes nacionales que soporten los esfuerzos individuales y comunitarios,

- fortalecimiento de las leyes y regulaciones que protejan la salud y la seguridad sanitaria,

- vinculación entre la comunidad y el personal de los servicios de salud y disponibilidad para la provisión de cuidados de salud cuando otras maneras no estén disponibles,

- garantía de una salud pública competente de la fuerza de trabajo del personal de cuidados de salud en los niveles nacionales, en términos de efectividad, accesibilidad y la calidad a nivel poblacional.

- generación de nuevas investigación sobre alternativas y soluciones innovadoras para controlar los riesgos para la salud(52)

La estrategia interprofesional en los niveles de grado y posgrado introducida en 2014se reconoce como una estrategia prometedora para mitigar la crisis de personal sanitario y mejorar la atención de salud que, si se lleva a cabo por equipos interprofesionales de salud, permite la optimización de las competencias y la prestación de asistencia sanitaria holística, de alta calidad y centrada en las personas. En diciembre de 2018, la OPS/OMS, en conjunto con le Secretaria de Gobierno de Salud de Argentina y la Red Regional de Educación Interprofesional(EIP) de las Américas realizaron la Tercera Reunión Técnica Regional "Educación Interprofesional en salud: Mejorar la capacidad de los recursos humanos para lograr la salud universal" donde se presentaron planes de acción de la estrategia de recursos humanos para el acceso universal a la salud y la cobertura universal de salud y su enfoque en la EIP. Sobre este marco, la OPS insta a los estados miembros a establecer iniciativas de acuerdo con sus necesidades y a promover equipos interprofesionales dentro de los servicios de salud, a través de la educación interprofesional. Se plantea desde esta iniciativa no solamente un ejemplo de traslacionalidad, sino también de intersectorialidad que insta a los sectores de educación y de salud a la construcción de estrategias conjuntas para la toma de decisiones políticas basadas en la mejor evidencia disponible y también a la indicción de nuevos conocimientos. $(52 ; 53)$

Este planteo refuerza el papel de la universidad en la responsabilidad que debe asumir en la formación de recursos para el desarrollo de salud para todos.

\section{Conclusiones}

En resumen en el dominio de la Salud para todos pueden identificarse ejes y atributos:

1. en cuanto a los espacios o dimensiones:

- $\quad$ se expresa en cooperación y relaciones internacionales.

- considera la interacción local/global.

2. en cuanto a los planos etiológicos:

- toma en cuenta las causas y las causas de las causas de la salud y las enfermedades, incluyendo los determinantes transnacionales como las migraciones, el cambio climático, las guerras y la seguridad alimentaria. 
3. en cuanto a intervenciones.

requiere participación y colaboración multi e interdisciplinaria e intersectorial; incorpora los cuidados clínicos basados en la evidencia científica ligada al contexto ya que entiende su práctica como una construcción social.

aborda el debate los conceptos de salud en relación con variables que intervienen en la toma de decisiones sanitarias: la información, la gobernanza, la diplomacia en salud y la seguridad.

4. El análisis desarrollado en los estudios de casos científicamente procesados permitirá: (a) identificar en todos los casos la validez metodológica de las intervenciones sanitarias desarrolladas y (b) reconocer las variables intervinientes en los diferentes escenarios en cada momento histórico-institucional.

\section{Bibliografía}

1. Heller M. Ciencia incierta. La producción social del conocimiento. $1^{\mathrm{a}} \mathrm{ed}$. Buenos Aires: Biblos; 2004. (pag 21) ISBN 950-786-415-4

2. Heller M. Ciencia incierta. La producción social del conocimiento. $1^{\mathrm{a} e d .}$ Buenos Aires: Biblos; 2004. (pag 54) ISBN 950-786-415-4

3. Garfinkel H. Studies in ethnomethodology. Englewood Cliffs, NJ Prentice Hall, 1967. Mencionado por Knorr Cetina K. La fabricación del conocimiento. Un ensayo sobre el carácter constructivista y contextal de la ciencia. Buenos Aires. U. N. de Quiles Editorial; 2008. (pag 98)

4. Bordoni N. Programas interdisciplinarios: Una mirada desde los problemas. En Universidad y Sociedad. Desafíos de la investigación interdisciplinaria. Buenos Aires: PIUBAMAS/Eudeba; 2014

5. Pagliai L; Bordoni N.Propuesta de indicadores de impacto social en investigación en salud pública. Documento para la autoevaluacion de los sistemas de investigación para países en desarrollo. Revista de la Maestría en Salud Pública. (2007) Año 5 No. 9. (visitado 10 marzo 2018) ISSN: 1667-3700

6. Busso G. Vulnerabilidad social: nociones e implicancias de políticas para Latinoamerica a inicios del siglo XXI. Santiago de Chile: CEPAL; 2001 (Cap III) Disponible en www.cepal.org $>\mathrm{xml}>$ gbusso

7. MacFarlane SB, Jacobs M, Kaaya EE. In the name of global health: trends in academic institutions. J Public Health Policy. 2008;29:383-401.

8. Koplan JP, Bond TC, Merson MH, Reddy KS, Rodriguez MH, Sewankambo NK, et al. Towards a common definition of global health. Lancet. 2009;373:1993-5.

9. Fried LP, Bentley ME, Buekens P, Burke DS, Frenk JJ, Klag MJ, et al. Global health is public health.Lancet. 2010;375:535-7.

10. Harrar L. Definitions of global health.The 2005 PBS series Rx for survivalıs approach. J Public Health Policy. 2008;29:402-3.

11. Fidler DP. After the revolution: global health politics in a time of economic crises and threatening future trends. Global HealthGovernance. 2009:2 disponible: http:// www.ghgj.org

12. Informe sobre el desarrollo mundial 2005: Un mejor clima para la inversión en beneficio de todos. Programa de las Naciones Unidas para el Desarrollo. WDR(DECWD); 2005. ISSN 0163-5085

13. Winslow C.E.A. The untilled fields of Public Health. Science .1920; 51:23-33

14. Frenk J. La salud pública: campo del conocimiento y ámbito para la acción. Salud Pública de Mexico. 1988; 30: 246 -54

15. Institute of Medicine. The Future of the Public's Health in the 21st century. Washington DC.: The National Academies Press; 2002

16. Birn AE, Fee E:The Rockefeller Foundation and the international health agenda. 
The Lancet.2013; 381(9878): 1618-9

17. Merson MH, Black RE, Mills AJ. International public health: diseases, programs, systems, and policies.2nd ed. Sudbury, MA: Jones and Bartlett; 2006.

18. Teruel JR. Los desafios de la salud internacional. Notas sobre su historia y retos previstos para el futuro. En OPS/OMS. Programa de Líderes en Salud Internacional Edmundo Granda Ugalde; 2010

19. Kickbush I. The need for a European strategy on global health. Scand J Public Health. 2006;34:561-5

20. Beaghole R, Yach D. Globalization and theprevention and controlof noncommunicable disease; The neglected chronic disease of adults. The Lancet 2003; 362: 903-8

21. European perspectives on global health: a policy glossary. En: European Foundation Centre. Brussels: European Foundation Centre; 2006.

22. HM Government. Health is global: a UK Government strategy 2008-13. London: UK Government; 2008

23. Pan American Health Organization. Strategy for Universal Access to Health and Universal Health Coverage [Internet]. En: 53rd Directing Council of PAHO, 66th Session of the Regional Committee of WHO for the Americas; 2014 Sep 29-Oct 3; Washington (DC): PAHO; 2014.(Official document CD53/5, Rev. 2) [cited May 26, 2017]. Disponible en: http://www.paho.org/hq/index.php?option=com docman\&task $=$ doc_download\&gid $=27273 \&$ Itemid $=270 \& l a n g=e s$

24. Teruel JR., Wegman M. Global Health.University of Michigan: Epidemiology; 1998

25. Davison C, Ndumbe-Eyoh S, Clement C. Critical examination of knowkege to accion models and implications for promoting health equity. International $\mathrm{J}$ for Equity in Health. 2015: 14:49 Disponible en http://dx.doi: 10: 1186/s1293901501787 ISSN 14759276

26. SenA K. Development as freedom. Oxford: Oxford University Press; New York: Alfred Knopf, 1999

27. Solar O, IrwinA . Social determinants, political context and civil society action: a historical perspective of the Commission on Social Determinants of Health. Health Promotion Journal of Australia.2006; 17 (3) 163: 180-5

28. Lopez A, Mathers CD, Ezzati M, Jamison DT, . Murray C .Selected major risk factors and global and regional burden of disease. TheLancet2002;360(9343):134760

29. Bordoni N. Formación de recursos humanos en salud para el desarrollo. Medicina y sociedad. 2014; julio (ISSN 1669-7782) Disponible en http://www.medicina y sociedad.org.ar/.

30. Bordoni N, SquassiA.”Vinculación entre la universidad y la sociedad: Experiencias de la Universidad de Buenos Aires". en Sanchez Medina J, Lopez Vidales R (Eds). "Promocion de salud escolar en Iberoamerica: experiencias". Documentos de promoción de salud escolar. Vol 1. Sevilla: Red iberoamericana de Promoción de Salud escolar. (RIPSE); 2012.

31. Souza Casadinho J. Dinámica de uso de los agrotóxicos y su relación con la salud socio ambiental. En Carrizo C, Berger M(comp).Justicia ambiental. El trabajo interdisciplinario en agrotóxicos y transgénicos. Córdoba : E-book; 2013 ISBN 978-987-33-3819-9.

32. Bordoni N. Nuevas tendencias en la vinculación entre la universidad y la sociedad. EnBordoni N, Escobar A, Castillo Mercado R.Odontología Pediátrica. La salud bucal del niño en el mundo actual. Buenos Aires: Médica Panamericana; 2010. ISBN 978-950-06-0119 (Cap

33. Buyayisqui MP, Bordoni N, Garbossa G. Overcoming Language and Cultural 
Barriers. A Graphical Communication Tool to Perform a Parasitological Screening in two Vulnerable Populations from Argentina. Journal of Health Communication, 0:1-13, epubeheadoct 2012. ISSN: 1081-0730 print / 1087-0415 online

34. Bordoni N. Estudio de un caso de integración entre la investigación, la docencia y la extensión en el escenario de la atención odontológica. En Riquelme, G. C. (editora) Sinergia pedagógica en universidades argentina: articulación de la docencia, la investigación, la extensión y la transferencia en el desarrollo de planes de estudio. Tomo III. Buenos Aires: Miño y Dávila Editores; 2009. ISBN 978-84-96571-88-2

35. Lucarelli E. Teoría y práctica en la Universidad . Buenos Aires: Miño y Davila; 2009 (Cap 4)

36. Garbossa G, Buyayisqui MP, Geffner L, López Arias LS, de la Fournière S, Haedo AS, Marconi A, Frid JC, Nesse A, Bordoni N. Social and environmentalhealthdete rminants. Water- and excreta-related parasitoses in a shantytown in Buenos Aires, Argentina. Pathogens and Global Health. 2013; 107: 141-52. ISSN 2047-7724.

37. Bordoni N. Impacto de las investigaciones sobre salud para el desarrollo: Papel de la universidad en la traslación de conocimientos a las decisiones político-sanitarias. Buenos Aires. PIUBAMAS/SeCyT: 2016. Disponible en www.uba.ar>archivos secyt>image.(pag 1-13)

38. Frenk J, Chen L, Bhutta Z, Cohen J, Crisp N, Evans T, Fineberg H, Garcia P, Ke Y, Kelley P, et al. (Education of Health Professionals for the 21th Century, A Global IndependientComission) Health Professionals for a New Century; transforming education to strengthen health systems in an interdependent world. The Lancet 2010; 376:1923-58.

39. Bordoni N y Squassi A. Las migraciones y la salud bucal en el marco de la salud global . Documento de posición. Rev de Salud Pública. F. Medicina UNC. 2016; dic DOI: $10.31052 / 1853.1180 . v 20 . n 3.14400$.

40. Rovere M Redes en salud. Un nuevo paradigma para el abordaje de las organizaciones y la comunidad. Rosario: Secretaria de Salud Pública de la Municipalidad de Rosario; 1999. Cap 2.

41. Welch V., Jill J, PetkavicJ et al. Protocol for the development of a CONSORTequity-guideline to improve reporting of health equity in randomized trials. Implement Sci 2015, 10: 146 .on line 2015 oct 2. Doi 10. 1186/s13012-015- 0332-2

42. Bordoni N. Programas interdisciplinarios: Una mirada desde los problemas. En Universidad y Sociedad. Desafíos de la investigación interdisciplinaria. Buenos Aires: PIUBAMAS/Eudeba ;2014.

43. Lomas J, Culyer T, McCutcheon C, McAuley L, Law S . Conceptualizing and combining evidence for Health. System Guidance. CanadianHealthServicesResea rchFoundation.Canadá. CHSRF, 2005

44. Editorial. Evaluation: the top priority for global health. Lancet, 2010; 375-526 [PubMed)

45. Conferencia Mundial sobre la Ciencia, 1999. Science for the Twenty-first Century . A New Commitment, Social Science Agenda Framework for Action. Budapest: UNESCO-ICSU

46. Harris P. Harris-Roxas B, Harris E, Kemp L. Health Impact Assesment: A practical guide. Sidney, CHETRE. UNSW Research Centre for Primary Haealth, Care and Equity. UNSW. 2007.

47. Sabatier PA. Top-dawn and bottom-upapproaches to implementation research. .J Pub Policy. 1986. 6: 21-48 42.

48. Nowotny H, Scott P Gibbons M. Rethinking science: knowledge in an age of uncertainty. Cambridge:Polity; 2001

49. Woolf SH, Purnell JQ, Simon SM, Zimmerman EB, Camberos GJ, Haley A, Fields RP.Translating evidence into population health improvement: strategies and 
barriers.Annu Rev Public Health. 2015 Mar 18;36:463-82. doi: 10.1146/annurevpublhealth-082214-110901. Epub 2015 Jan 12

50. ONU. International Forum on the erradication of poverty. Department of Economics and Social Affairs. UnitedNationsHeadquarters , 15/16 november; 2006

51. UNESCO. Gestión de las transformaciones sociales (MOST). Programa intergubernamental de la UNESCO. UNESDOC: París; 2001

52. Bordoni N. La extensión como objeto de investigación. Análisis desde el campo de la salud. Revista de Extensión universitaria. 2018. (9): Investigación y extensión universitaria/Desafíos desde la gestión- DOI: https://doi.org/10.14409/extension. v819.Jyl-Dic 7852

53. Mikael SSE, Cassiani SHDB, Silva FAM. The PAHO/WHO Regional Network of Interprofessional Health Education. Rev. Latino-Am. Enfermagem. 2017;25:e2866. [visitado: 05/03/19. Disponible en DOI: http://dx.doi.org/10.1590/15188345.0000 .2866 